\title{
Hiperplasia linfoide nodular
}

\section{Nodular lymphoid hyperplasia}

\author{
Julián Ñáñez, Fredy Calambas, Adrián Maya • Popayán (Colombia)
}

\section{Resumen}

La hiperplasia linfoide nodular es una patología poco frecuente, con mayor prevalencia en la población pediátrica; sin embargo, también afecta a los adultos. Se caracteriza por la presencia de múltiples nódulos pequeños, normalmente entre 2 y $10 \mathrm{~mm}$ de diámetro, distribuidos a lo largo del intestino delgado (más a menudo), estómago, intestino grueso o recto. Esta patología se ha documentado como respuesta a diferentes tipos de estímulo, por lo general infeccioso como VIH/ síndrome de inmunodeficiencia adquirida, giardiasis, infección por Helicobacter pylori, enfermedad celiaca y, en casos específicos, se acompaña de la enfermedad inflamatoria intestinal. El cuadro clínico no es específico y en muchas oportunidades es un hallazgo incidental, no tiene un tratamiento particular y se debe vigilar por el riesgo asociado a linfoma.

Palabras clave: hiperplasia linfoide nodular $(H L N)$, endoscopia gastrointestinal, tracto gastrointestinal, adultos, endoscopia, linfoma.

\begin{abstract}
Nodular lymphoid hyperplasia is a rare pathology, with a higher prevalence in the pediatric population; however,it also affects adults. It is characterized by the presence of multiple small nodules, usually between 2 and $10 \mathrm{~mm}$ in diameter, distributed throughout the small intestine (more often), stomach, large intestine or rectum. This pathology has been documented as a response to different types of stimuli, usually infectious such as HIV / acquired immune deficiency syndrome, giardiasis, Helicobacter pylori infection, celiac disease and, in specific cases, is accompanied by inflammatory bowel disease. The clinical picture is not specific and in many opportunities is an incidental finding, has no particular treatment and should be monitored because of the risk associated with lymphoma.
\end{abstract}

Key words: nodular lymphoid hyperplasia $(N L H)$, gastrointestinal endoscopy, gastrointestinal tract, adults, endoscopy, lymphoma.
Dr. Julián Ñáñez Paz: Especialista en Medicina Interna; Dr. Fredy Calambas: Especialista en Medicina Interna y Gastroenterología; Dr. Adrián Maya Rodríguez: Residente Medicina Interna. Universidad del Cauca, Hospital Universitario San José de Popayán. Popayán (Colombia).

Correspondencia: Dr. Adrián Maya Rodríguez, Popayán (Colombia).

E-mail: adrianmaya586@hotmail.com

\section{Introducción}

Hiperplasia linfoide nodular (HLN) es un estado linfoproliferativo raro que no es patognomónica de una enfermedad específica (1). El diagnóstico en algunas oportunidades es incidental y se basa en hallazgos patológicos de folículos hiperplásicos linfoides, centros germinales mitóticamente activos con mantos linfocíticos bien definidos y los folículos linfoides localizados en la mucosa o submucosa. Su causa es desconocida, pero se asocia por lo general con un estado de inmunocompromiso que incluyen: quimioterapia, infección viral de la inmunodeficiencia adquirida y trasplante de órganos. La HLN se asocia frecuentemente con cualquiera de varias enfermedades benignas o malignas en adultos (3), es importante dilucidar entre NLH benigna y linfoma maligno.

\section{Reporte del caso}

Paciente masculino de 42 años, procedente de zona urbana de Popayán (Cauca), trabajó como escolta hasta hace cinco años. Tiene antecedentes de alergia con manifestaciones cutáneas al tramadol, diclofenaco, dipirona y rash cutáneo ocasional; antecedente de sinusitis aguda maxilar izquierda y antecedente familiar de padre que falleció por linfoma a la edad de 56 años.

Cuadro clínico de 20 años de evolución que inició con la aparición de deposiciones diarreicas mucoides asociadas a episodios de hematoquecia y dolor en hemiabdomen derecho, además, presentó aparición de debilidad, palpitaciones, sudoración, fiebre vesperal, disminución de $6 \mathrm{Kg}$ en 10 meses, artralgias generalizadas, calambres en miembros inferiores y prurito generalizado; al examen físico se destacó 
la presencia de exoftalmos, eritema ocular simétrico, escasas adenopatías cervicales posteriores blandas no adheridas a planos profundos y marcado dolor a nivel del hemiabdomen derecho. En varias oportunidades recibió antibiótico con metronidazol y tetraciclinas sin mejoría.

Examen físico: alerta, sin alteración en los signos vitales, ECOG 2 (Eastern Cooperative Oncology Group) (2), peso: $92 \mathrm{Kg}$, talla: $180 \mathrm{~cm}$, alerta, sin adenopatías; abdomen: cicatriz de apendicectomía y leve dolor a la palpación en fosa iliaca derecha, sin esplenomegalia; puntos gatillo para fibromialgia (todos los puntos son dolorosos).

El resultado de los coprológicos seriados mostraron deposiciones cafés blandas con flora bacteriana aumentada, ocasionalmente con blastocystis hominis y tinción de Wright negativa; tránsito intestinal evidenció dilatación de asas medias y distales asociadas a engrosamiento de las válvulas conniventes. La tomografía abdominal reveló múltiples adenopatías a nivel mesentérico, con diámetro transverso de $1 \mathrm{~cm}$, y escasas retroperitoneales con calcificación endoluminal a nivel del apéndice cecal. Ante la sospecha de linfoma, el hematólogo realizó aspirado de médula ósea observándose normocelular con representación normal de todas las series salvo una leve hiperplasia de eosinófilos, con cambios megaloblásticos moderados que comprometía sobre todo la línea mieloide, consistente en bandas, metamielocitos y evidencia de asincronismo en la maduración de la serie roja sin observarse blastos ni otras anormalidades; así mismo, estudios complementarios descartaron infecciones virales, bacterianas, disfunción tiroidea o condiciones autoinmunes; la gammagrafía de tiroides fue normal, el ecocardiograma transtorácico reveló discreta hipertrofia ventricular izquierda sin otras alteraciones estructurales y la fracción de eyección conservada del 60\%; la electromiografía y la velocidad de conducción nerviosa descartó la presencia de neuropatía periférica.

La colonoscopia reportó pérdida del patrón mucoso normal por presencia de múltiples nódulos y pólipos de aspecto inflamatorio con patrón en "empedrado" a nivel del íleon terminal asociado a escasas erosiones hiperémicas difusas a nivel del colon sigmoide; las biopsias de estas lesiones mostraron la presencia de múltiples folículos linfoides hiperplásicos que comprimían las glándulas de la mucosa generando aspecto polipoide, con atrofia de la lámina propia e infiltrado inflamatorio linfoplasmocitario sugiriendo ileítis terminal por enfermedad de Crohn y, menos probable tuberculosis intestinal. La biopsia de sigmoide fue normal. Por lo anterior, se inició tratamiento con budesónida $9 \mathrm{mg}$ /día y azatioprina $100 \mathrm{mg} /$ día en el contexto de un cuadro de enfermedad inflamatoria intestinal. En la Tabla 1 se presenta el resumen de laboratorios, en la Figura 1 la enteroscopia, y en la Figura 2 la biopsia del íleon.

El paciente es valorado en consulta externa por gastroenterología, encontrando estudios no concluyentes con patología estudiada; sin embargo, no hay cohrencia con resultados antiguos por lo que se decide iniciar estudio para inmunodeficiencia común variable o asociada a IgA. Los resultados de inmunoglobulinas fueron normales: $\operatorname{IgE}$ $3,5 \mathrm{mg} / \mathrm{dL}$, IgM $554 \mathrm{mg} / \mathrm{dL}$, IgG $971 \mathrm{mg} / \mathrm{dL}, \operatorname{Ig}$ A $177 \mathrm{mg} /$ dL normales. Además, se solicitó la revisión de placas más inmunohistoquímica: yeyuno normal, duodenitis, hiperplasia nodular linfoide; inmunohistoquímica: infiltrado linfoide prominente en la lámina propia de ambas muestras, forma folículos linfoides que expresan CD20, CD10 y el BCL-6 son positivos en los linfocitos de centros germinales, los CD3 se expresan en los linfocitos T interfoliculares, CD23 positivo en células dendríticas de los folículos linfoides, el BCL- 2 positivo en los linfocitos $\mathrm{T}$ y en los linfocitos $\mathrm{B}$ de la zona del manto y negativo en los centros germinales; $\sin$ expresión de ciclina D1, cabe anotar que el índice de proliferación $\mathrm{Ki}-67$ es alto en los centros germinales como ocurre en los procesos reactivos no neoplásicos, concluyendo una hiperplasia nodular linfoide y una ileítis crónica severa, por lo tanto, al no documentarse malignidad se da salida por hematooncología. En el momento se encuentra estable y se debe continuar la vigilancia, sin embargo, hasta el momento no ha presentado recaída clínica.

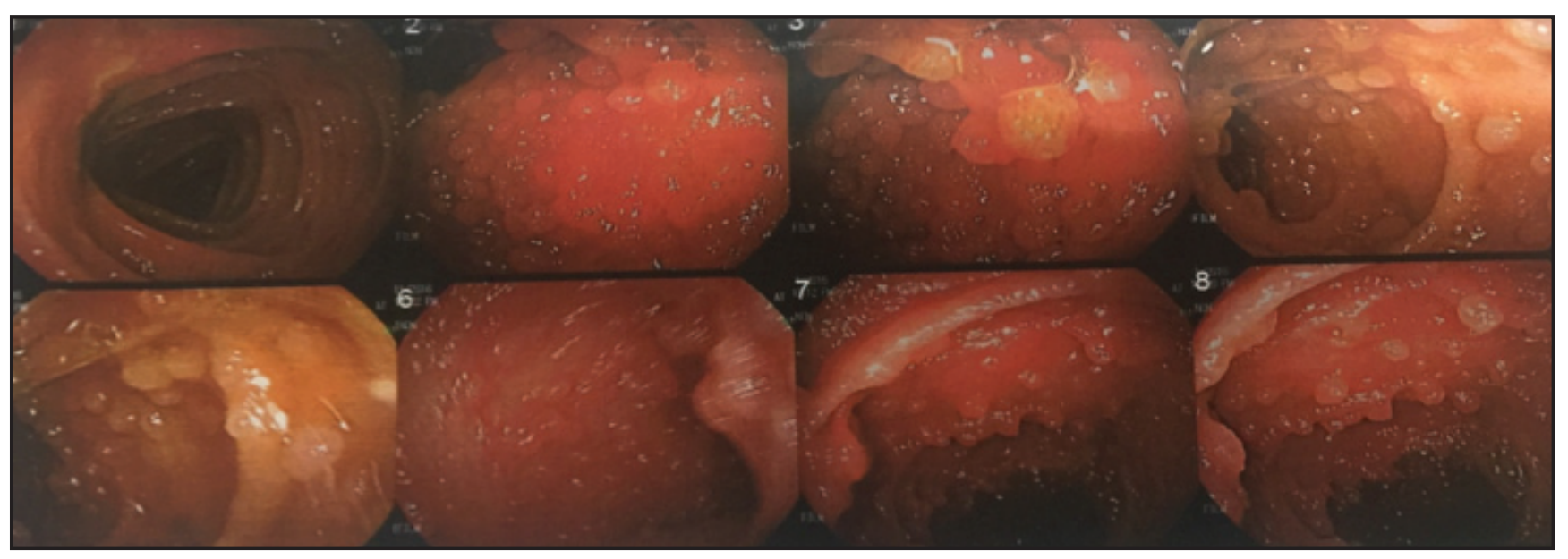

Figura 1. Enteroscopia: aspecto endoscópico de la mucosa del Ilion porción superior. 
Tabla 1. Paraclínicos

\begin{tabular}{|c|c|}
\hline \multicolumn{2}{|c|}{ Examen de laboratorio } \\
\hline Leucocitos & $\begin{array}{c}7.000 \text { células } / \mathrm{mm}^{3}(4.500- \\
10.000)\end{array}$ \\
\hline Eosinófilos & $3.2 \%(0-4)$ \\
\hline Hemoglobina & $15 \mathrm{~g} / \mathrm{dL}(14-18)$ \\
\hline Hematocrito & $47 \%(42-54)$ \\
\hline Plaquetas & $\begin{array}{c}232.000 \times 10^{3} / \mathrm{mm}^{3}(140- \\
400)\end{array}$ \\
\hline Proteína $\mathrm{C}$ reactiva & $<6 \mathrm{UI} / \mathrm{ml}$ \\
\hline VSG & 35 mm/hora $($ Menor < 15) \\
\hline Glucemia & $93 \mathrm{mg} / \mathrm{dL}(70-110)$ \\
\hline Creatinina sérica & $0.7 \mathrm{mg} / \mathrm{dL}(0.7-1.4)$ \\
\hline Albúmina & $4.4 \mathrm{~g} / \mathrm{dL}(3.1-5.0)$ \\
\hline Fosfatasa alcalina & $178 \mathrm{U} / \mathrm{L}(30-130)$ \\
\hline Aspartato aminotranspeptidasa (TGO) & $26 \mathrm{U} / \mathrm{L}(0-45)$ \\
\hline Alanino aminotransferasa (TGP) & $31 \mathrm{U} / \mathrm{L}(10-50)$ \\
\hline $\mathrm{LDH}$ & $528 \mathrm{U} / \mathrm{L}$ \\
\hline Beta 2 microglobulina sérica & $3.9 \mathrm{mg} / \mathrm{L}(1.2-2.8)$ \\
\hline Linfocitos CD4 & 543 células/ul (400-1500) \\
\hline Linfocitos CD8 & 336 células/ul $(150-1050)$ \\
\hline Linfocitos CD3 & 879 células/ul \\
\hline Relación CD4 / CD8 & $1.6(0.9-1.8)$ \\
\hline Prueba de tuberculina & Negativa \\
\hline IgG anti - citomegalovirus & 20.4 (Reactivo $>11)$ \\
\hline IgG anti - Epstein Barr & 30.6 (Positivo > 11) \\
\hline IgG anti - toxoplasma & Negativa $(\operatorname{IgG}<1: 16)$ \\
\hline $\begin{array}{l}\text { Venereal Diagnosis Research Lab } \\
\text { (VDRL) }\end{array}$ & No reactiva \\
\hline Anti - HIV & Negativo \\
\hline Antígenos febriles & Negativos \\
\hline
\end{tabular}

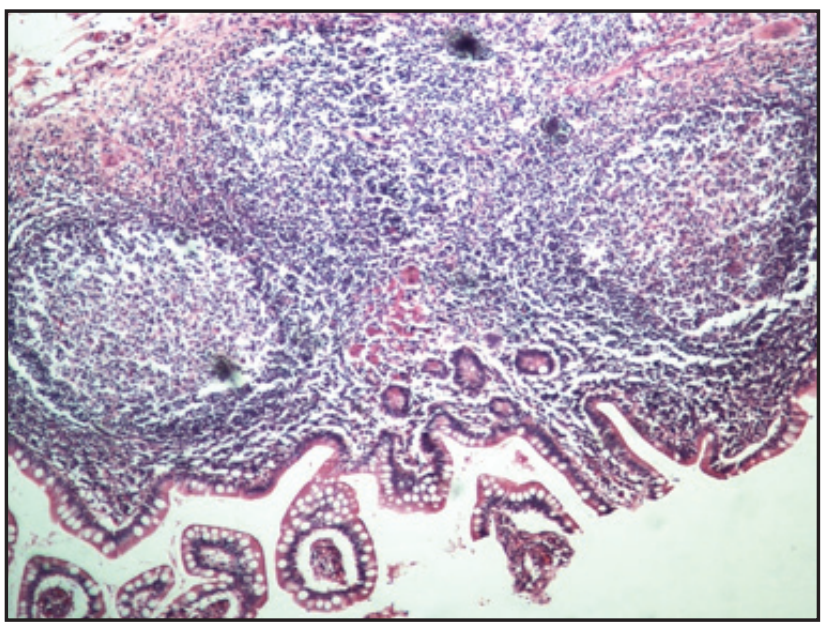

\section{Discusión}

la hiperplasia linfoide nodular (HLN) se caracteriza por la presencia de múltiples nódulos pequeños, entre 2 y $10 \mathrm{~mm}$ de diámetro, puede comprometer el íleon terminal principalmente, pero también puede encontrarse en el estómago, colon y recto (1). Histológicamente, se define por centros germinales marcadamente hiperplásicos, mitóticamente activos y bien definidos linfocitos en la lámina propia o en la submucosa superficial (3).

Hay diferentes hipótesis para explicar este hallazgo, en varias oportunidades es un hallazgo incidental, sin embargo, se ha descrito que en personas inmunocomprometidas podría representar un mecanismo compensatorio a la pobre respuesta inmunológica en el tracto digestivo (4), mientras que en inmunocompetentes podría ser secundaria a sobre estimulación del tejido linfoide asociado con el tubo digestivo por estímulos nocivos a la mucosa, como es el caso de la enfermedad inflamatoria intestinal. Para algunos autores se considera un estadio transicional y un factor de riesgo para la aparición de linfomas intestinales (5-10).

La incidencia es desconocida; sin embargo, HLN se considera una afección rara en adultos (1-4), esta ha sido asociada con varias enfermedades inmunodeficientes como la inmunodeficiencia común variable, la deficiencia selectiva de IgA, algunas enfermedades de tipo infeccioso como el VIH/SIDA, la infección por Helicobacter pylori y giardiasis (6-12). El cuadro clínico varía según la edad, en los niños se ha asociado a obstrucción intestinal e intususcepción, es muy rara y por ello se describe principalmente en esta población, en los adultos el dolor abdominal es crónico, también produce hemorragia gastrointestinal ya sea hematoquecia o melenas (4).

Este caso clínico se presenta en un paciente inmunocompetente al que se le descartó patologías infecciosas descritas como probables agentes etiológicos de esta patología, evidenciando como el diagnóstico clínico e histológico pueden llegar a un resultado positivo siempre y cuando se realicen de

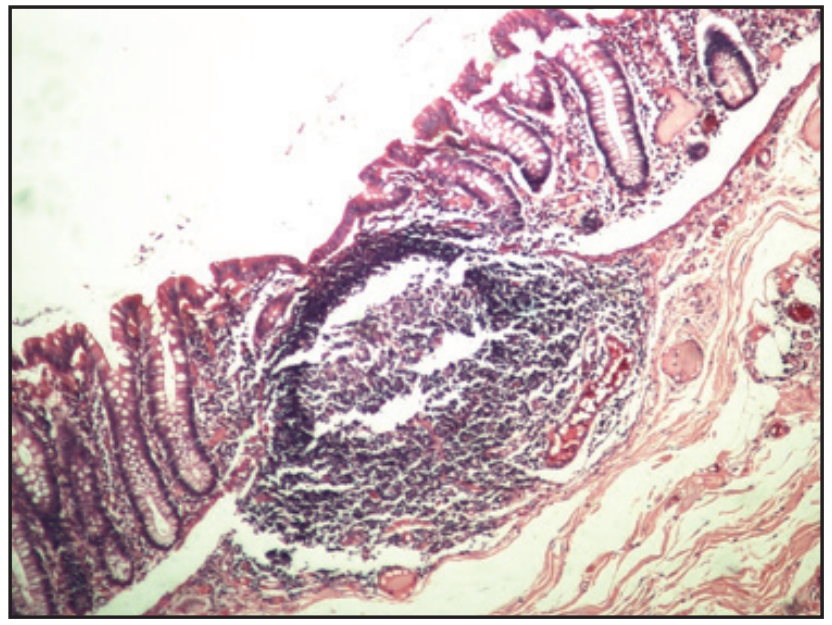

Figura 2. Biopsia del Íleon: coloración de eosina hematoxilina se observa fragmentos del ilion con folículos linfoides hiperplásicos $100 x$ CDO. 
forma ordenada, coherente con las diferentes especialidades; si bien, el estudio histopatológico es el "Gold Standard" (4) para esta patología, es necesario determinar y estar seguro que no estamos frente a un proceso neoplásico en curso, ya que guarda similitudes tanto en la endoscopia como en la histología, de ahí la necesidad de llegar a la realización de la inmunohistoquímica para aclarar si el paciente está carente de malignidad (9).

A la luz de la evidencia, es muy poco lo que se conoce de esta enfermedad y se puede decir que estamos ampliando el conocimiento al reportar estos casos, no hay guías, escasas revisiones de tema (4), sin embargo, el seguimiento a estos pacientes nos va a aportar si estamos o no frente a una lesión premaligna (11-12). En este paciente, no está asociado a otra enfermedad de base intestinal, sin embargo, es un objetivo disminuir la dosis de mesalazina a diferencia del caso reportado por Benedetti et al, donde la HLN se asoció a colitis (13).

La limitación más importante para este caso es la dificultad para disponer de todos los recursos tanto humanos como tecnológicos, las dificultades administrativas de las EPS, que llevaron a que pasarán varios años, hubo demora para la autorización y posterior realización de los diferentes estudios histológicos como de imagen; sin embargo, la buena articulación de las diferentes instituciones y profesionales ha permitido un buen desenlace tanto clínico como anímico para el paciente.

Si bien el tratamiento va dirigido a la condición asociada, es necesario continuar con una vigilancia periódica a pesar de que a la luz de la evidencia no se ha establecido el mejor intervalo de tiempo, ni método de seguimiento para la misma, en efecto existe el reporte de la utilización de la cápsula endoscópica para vigilar los cambios de riesgo de transformación maligna (12) y el advenimiento tecnológico de la F-fluoro-2-desoxiglucosa tomografía por emisión de positrones (PET-FDG) que se basa en el aumento de la glicolisis de las células cancerosas, siendo este el agente análogo de glucosa radiactiva FDG, que está atrapado dentro de la célula metabólicamente después de la fosforilación (9); por lo anterior, se debe investigar más a fondo si es PET o PET / CT pudieran sustituir la biopsia y, así, diferenciar entre hiperplasia linfoide benigna y linfoma maligno (8).

\section{Referencias}

1. Ranchod M, Lewin KJ, Dorfman RF. Lymphoid Hiperplasia of the Gastroentestinal Tract. A Study of 26 cases and Review of the Literatura. Am J Surg Pathol 1978; 2(4): 383 - 400.

2. Oken MM, Creech RH, Tormey DC, Horton J, Davis TE, McFadden ET, et al. Toxicity And Response Criteria Of The Eastern Cooperative Oncology Group. Am J Clin Oncol 1982; 5:649-55.

3. Rubio-Tapia A, Hernández-Calleros J, Trinidad-Hernández S, Uscanga L. Clinical characteristics of a group of adults with nodular lymphoid hyperplasia: a single center experience. WJG 2006;12(12):1945-8

4. Albuquerque A. Nodular lymphoid hyperplasia in the gastrointestinal tract in adult patients: A review. World J Gastrointest Endosc 2014;6(11):534-40.

5. Ward EM, Wolfsen HC. Review article: the non-inherited gastrointestinal polyposis syndromes. Alimentary pharmacology \& therapeutics 2002;16(3):333-42.

6. Kokkonen J, Karttunen TJ. Lymphonodular Hyperplasia on the Mucosa of the Lower Gastrointestinal Tract in Children: An indication of enhanced immune response? Journal of pediatric gastroenterology and nutrition 2002;34(1):42-6.

7. Iacono G, Ravelli A, Di Prima L, Scalici C, Bolognini S, Chiappa S, et al. Colonic Lymphoid Nodular Hyperplasia in Children: Relationship to Food Hypersensitivity. Clinical gastroenterology and hepatology: The official clinical practice journal of the American Gastroenterological Association. 2007;5(3):361-6.

8. Chung - Cheng L, Hsiang - Yao S, Chao-Hung K, Huang - Ming H, Yu Wen Ch, Deng-Chyang W, et al. Nodular lymphoid hyperplasia in endoscopic and FDG-PET/CT (18F-fluoro-2-deoxyglucose positron emission tomography/ computerized tomography) imaging. KJMS. 2013;29(7):396-99.

9. Monsanto P, Lérias C, Almeida N, Lopes S, Cabral JE, Figueiredo P, et al. Intestinal Nodular Lymphoid Hyperplasia and Extraintestinal Lymphoma-a Rare Association. Acta gastroenterol, Bélgica. 2012;75(2):260-2.

10. Chiaramonte C, Glick Seth N. Nodular Lymphoid Hyperplasia of the Small Bowel Complicated by Jejunal Lymphoma in a Patient with Common Variable Immune Deficiency Syndrome. Am J roentgenol. 1994;163(5):1118-19.

11. Luzi G, Zullo A, Lebba F, Rinaldi V, Sanchez Mete L, Muscaritoli M, et al. Duodenal Pathology and Clinical Immunological Implications in Common Variable Immunodeficiency Patients. American J Gastroenterol. 2003;98(1):118-21.

12. Postgate A, Despott E, Talbot I, Phillips R, Aylwin A, Fraser C. An unusual cause of diarrhea: diffuse intestinal nodular lymphoid hyperplasia in association with selective immunoglobulin A deficiency (with video). Gastroint Endosc. 2009;70(1):168-9.

13. Benedetti I, Hoyos J, Carmona R. Lymphoid Nodular Hyperplasia Associated with Ulcerative Colitis: Case Study and Literature Review. Rev Col Gastroenterol 2016; 31: $288-91$. 